\title{
Chemo-Profiling of Illicit Amphetamine Tablets Seized from Jazan, Saudi Arabia, Using Gas Chromatography-Mass Spectrometry and Chemometric Techniques
}

\author{
Hassan A. Alhazmi $\left(\mathbb{D},{ }^{1,2}\right.$ Waquar Ahsan $\mathbb{D}^{2},{ }^{2}$ Mohammed Al Bratty $\mathbb{D}^{2},{ }^{2}$ Asaad Khalid $\left(\mathbb{D},{ }^{1}\right.$ \\ Shahnaz Sultana $\mathbb{D}^{3},{ }^{3}$ Asim Najmi $\left(\mathbb{D},{ }^{2}\right.$ Hafiz A. Makeen $\mathbb{D}^{1},{ }^{4}$ Ibraheem M. Attafi $\mathbb{D}^{5}{ }^{5}$ \\ Farid M. Abualsail $\mathbb{D}^{5},{ }^{5}$ Mohammed A. Arishy $\mathbb{D D}^{5}$ and Ibrahim A. Khardali ${ }^{5}{ }^{5}$ \\ ${ }^{1}$ Substance Abuse and Toxicology Research Centre, Jazan University, P. Box No. 114, Jazan, Saudi Arabia \\ ${ }^{2}$ Department of Pharmaceutical Chemistry, College of Pharmacy, Jazan University, P. Box No. 114, Jazan, Saudi Arabia \\ ${ }^{3}$ Department of Pharmacognosy, College of Pharmacy, Jazan University, P. Box No. 114, Jazan, Saudi Arabia \\ ${ }^{4}$ Department of Clinical Pharmacy, College of Pharmacy, Jazan University, P. Box No. 114, Jazan, Saudi Arabia \\ ${ }^{5}$ Poison Control and Medical Forensic Chemistry Center, General Directorate of Health Affairs, Jazan, Saudi Arabia
}

Correspondence should be addressed to Waquar Ahsan; wmohammad@jazanu.edu.sa

Received 5 August 2021; Accepted 23 September 2021; Published 6 October 2021

Academic Editor: Lucica Barbes

Copyright (c) 2021 Hassan A. Alhazmi et al. This is an open access article distributed under the Creative Commons Attribution License, which permits unrestricted use, distribution, and reproduction in any medium, provided the original work is properly cited.

\begin{abstract}
A number of illegal drug tablets with unknown constituents are supplied to countries around the world, most of which are habit forming. Amphetamine constitutes the majority of illegal tablets supplied to Saudi Arabia. In this study, we investigated illicit amphetamine tablets seized from Jazan region located in the southwest of Saudi Arabia to identify the insidious additives present in them and their health-related risks. Tablets were analyzed for the presence of amphetamine and other additives using gas chromatography-mass spectroscopy (GC-MS) technique. Amphetamine was detected in good to high area \% values in all analyzed tablets in the range of $16.29-41.23 \%$. Interestingly, a number of other additives were also detected with amphetamine in most of the tested samples including caffeine, lidocaine, diphenhydramine, and 8-chlorotheophylline in considerable area \%. Caffeine may have been added to enhance the psychotic effect of amphetamine, whereas lidocaine was added to prevent the cardiovascular side effects of amphetamine. Diphenhydramine was probably added to prevent other undesirable side effects of amphetamine such as insomnia and tremors. Chemometric hierarchical cluster analysis was carried out to make samples clusters which have similar characteristics. It resulted into a dendrogram tree showing eight clusters signifying different sources of tablet samples. Owing to the toxic effects of amphetamine and other psychoactive constituents in the tested tablets, the illegal trafficking of these tablets should be prevented by all means and public awareness should be increased.
\end{abstract}

\section{Introduction}

Amphetamine is the psychostimulant drug that had been indicated clinically for the treatment of attention-deficit hyperactivity disorder (ADHD). However, it is no longer used clinically due to its addictive and other adverse effects. Nevertheless, amphetamine and its congeners are still produced and used illegally for their transient euphoric effects leading to mood elevation and increased physical and mental performance $[1,2]$. These drugs are known to show dose-dependent adverse effects on humans such as confusion, hallucinations, tremors, hypertension, and arrhythmias. Amphetamine tablets are generally supplied under the common brand name "captagon" containing the active ingredient "fenethylline hydrochloride," which is the theophylline derivative of amphetamine and has similar effects to those of amphetamine. Captagon tablets are believed to be originated from European countries and are widely 
consumed in Middle Eastern countries including Saudi Arabia, Kuwait, Jordan, and Qatar [3]. News regarding seizure of captagon tablets are very frequent in these countries. Instead of religious and legal prohibitions in Middle Eastern countries including Saudi Arabia, the use of narcotic substances is prevalent [4]. It was estimated in 2019 that around $7-8 \%$ of Saudis are drug users and $70 \%$ among them are of age 12-22 years $[5,6]$. Also, the accuracy of this data is not clear and the United Nations Office on Drugs and Crime (UNODC) suggests that this data may be underestimated owing to the higher number of seizures in these areas. The social stigma and fear of disclosure associated with the use of these substances further decrease the numbers. It was also estimated that more than $50 \%$ of the total global interception of amphetamine-type stimulants (ATS) is reported from the Arabian Peninsula [7].

A number of cases of amphetamine tablet seizures have been reported worldwide earlier and were analyzed for their content using various analytical techniques. Results revealed that amphetamine was present in varied quantities in different tablets, from trace amounts to even more than onethird of the total weight of the tablets. Previously, analysis of several seized captagon tablets from Saudi Arabia showed presence of amphetamine in trace quantities in almost half of the tablets; however, the other half showed very high concentration of more than 38\% [8]. Similarly, another seizure from Iraq by the Drug Enforcement Administration (DEA) showed that the tablets contained varying concentrations of amphetamine (7-20 mg) along with several other additives. These included caffeine $(30-65 \mathrm{mg})$, theophylline (14-39 mg), and acetaminophen (9-21 mg) [9]. Similar results were obtained when German Bundeskriminalamt (BKA) carried out the analysis of seized tablets in 2013 and reported the presence of amphetamine (8-14\%) along with other additives including caffeine (12-35\%), theophylline (10-14\%), and acetaminophen (6-20\%) [10].

A number of tablets of unknown constituents were detained from three major cities of Jazan, Saudi Arabia, which were suspected to be containing amphetamine. Earlier, they were subjected to the LC-MS/MS analysis by our research group at the Poison Control and Medical Forensic Chemistry Center, Jazan, to determine the concentration of amphetamine and methamphetamine in these tablets. The amounts of amphetamine in the tested tablets were calculated to be between 9.07 and $14.77 \mathrm{mg}$, which were considerably high [11]. In continuation to the analysis of these tablets for the determination of unknown constituents, we intended to perform the GC-MS analysis of these tablets which could give information of all constituents present in the tablets. Previously, we successfully employed techniques such as GC-MS and ICP-MS to perform analysis of various other substances of abuse and determined a number of constituents [12-15] in their samples. Analysis of amphetamine tablets seized in Jazan province is very important keeping in consideration the fact that Jazan province is situated at the southwest of Saudi Arabia and shares borders with Yemen where the use of amphetamine is common. The similarities in constituents would give an insight about the place of origin of these tablets. No previous study has been reported to investigate the constituents of seized amphetamine tablets in this region.

\section{Materials and Methods}

2.1. Chemicals and Reagents. The standard amphetamine and the internal standard (IS) amphetamine-D5 vials (1 mg/ $\mathrm{mL}$ ) were procured from Lipomed AG (Cambridge, USA). All other chemicals and solvents including isopropyl alcohol, ammonium hydroxide, dichloromethane, methanol, hexane, ethyl acetate, acetonitrile, and acetic acid were purchased from Sigma-Aldrich (Steinheim, Germany). All the solvents used were of HPLC grade. Extrapure Deionized (DI) water was prepared in our lab using Millipore purification system.

2.2. Sample Collection. Sample tablets were seized and collected by local police raid from Ahad Al-Masariha, AlDarb, and Jazan cities of Jazan province and sent to the Poison Control and Medical Forensic Chemistry Center, General Directorate of Health Affairs, Jazan, for further analysis. For the determination of chemical constituents, randomly 10 tablets seized from each city were selected. Coding of samples was performed according to the city from where they were collected: A1-A10 (Ahad Al-Masariha), D1D10 (Al-Darb), and G1-G10 (Jazan/Gizan city). All these thirty tablets were analyzed separately using the developed GC-MS method at the Poison Control Center.

2.3. Preparation of Solutions. Amphetamine standard solution $(1 \mathrm{mg} / \mathrm{mL})$ was further diluted appropriately using methanol to obtain the solutions of concentrations $5,10,15$, 20 , and $25 \mu \mathrm{g} / \mathrm{mL}$ which were used to construct the calibration curve. The IS amphetamine-D5 solution was added to each calibration standard keeping the IS concentration at $10 \mu \mathrm{g} / \mathrm{mL}$. Three quality-control (QC) solutions of concentrations of 5 (low; LQC), 10 (medium; MQC), and $20 \mu \mathrm{g} /$ $\mathrm{mL}$ (high; HQC) were also prepared using the standard stock solution of amphetamine. An aliquot of $2 \mathrm{~mL}$ from each calibration standard was placed into the GC-vial and $2 \mu \mathrm{L}$ was injected to the system and the analysis was performed in triplicate.

2.4. Sample Preparation. Half tablet from each sample was crushed into fine powder and dissolved in $3 \mathrm{~mL}$ methanol and sonicated using WUC.D10H digital ultrasonicator (Acinterlab, Fl, USA) for $15 \mathrm{~min}$ at room temperature [16]. Samples were then filtered to the GC-vial using $0.45 \mu \mathrm{m}$ Millex-LCR PTFE syringe filter (Merck Millipore, MA, USA) and the analyses were performed in triplicate. Samples were prepared fresh before each analysis.

2.5. Method Validation. The SPE method for sample preparation as well as the proposed GC method was validated according to the ICH guidelines Q2B. The intraday and interday precisions were determined by calculating the $\%$ relative standard deviation (\% RSD) of peak areas of amphetamine standard and measuring the retention times of 
the three replicate injections. Accuracy of the experiment was determined by calculating the \% recovery of the amphetamine. The quality control samples $(5,10$, and $20 \mu \mathrm{g} /$ $\mathrm{mL}$ ) were analyzed to get the data for precision and accuracy. All the quality control samples were analyzed on the same day to get the intraday precision and accuracy data, whereas the samples were analyzed for three consecutive days to get the interday data.

Linearity of the proposed method was also evaluated using the ICH protocol by analyzing the above prepared calibration standards. The prepared standard solutions of five concentrations were injected in increasing concentrations and the chromatograms were recorded. The corresponding peak area values were calculated and the calibration curve was plotted between the peak area ratio of amphetamine standard and amphetamine-D5 internal standard against amphetamine concentration and the intercept and slope values were obtained from the plot. For the determination of sensitivity of the method, the limit of detection (LOD) and limit of quantification (LOQ) values were calculated from the standard deviation and slope of the curve.

2.6. GC-MS Analysis of Tablets. Shimadzu Gas Chromatograph (Shimadzu, Japan) was used for GC-MS analysis for all the samples and solutions. The GC instrument was equipped with a TR-5MS capillary column $(30 \mathrm{~m} \times 0.25 \mathrm{~mm})$ having a $0.25 \mu \mathrm{m}$ film thickness. The carrier gas helium $(\mathrm{He})$ was used at a flow rate of $1.2 \mathrm{~mL} / \mathrm{min}$ throughout the process. Instrument was heated to $70^{\circ} \mathrm{C}$ initially, followed by ramping at a rate of $15^{\circ} \mathrm{C}$ per min to $300^{\circ} \mathrm{C}$, and held for $30 \mathrm{~min}$. Fragments were detected using Shimadzu QP2010 Ultra MS detector coupled to the chromatograph instrument with electron-ionization (EI) system and working at an ionization energy of $70 \mathrm{eV}$. The temperature of the ion source was kept at $230^{\circ} \mathrm{C}$. The mass spectrometric analysis was performed by obtaining a full mass spectrum over a range of $\mathrm{m} / \mathrm{z} 100-550$ and $m / z 136$ and 141 were used for monitoring amphetamine and amphetamine-D5, respectively. All the analyses were carried out in triplicate.

2.7. Identification of Constituents. The constituents present in the samples were identified by using authentic standards (Sigma Aldrich, Steinheim, Germany) present in our laboratory and by comparing their retention indices. The major components present in the tablets were identified using the authentic standards of amphetamine, lidocaine, and caffeine present in our laboratory. The retention indices and the fragmentation pattern which were in close agreement to reference standards were used for identification. For other constituents, the fragmentation patterns were compared and identified using built-in NIST08 and Wiley 9 libraries stored in the software database. The area $\%$ values $( \pm S D)$ were used for quantification of the constituents.

2.8. Chemometric Analysis. Owing to the significant variations in the constituents of tablets, most abundant constituents were selected and subjected to multivariate chemometric analysis in order to determine the relation, if any, among the constituents. Hierarchical Cluster Analysis (HCA) was performed and dendrogram trees were obtained using NCSS software 2020 version. The area \% values of different constituents were considered as variables and were expressing columns, whereas the samples constituted 30 rows. The HCA analysis was run to obtain significant groups of samples or clusters which have similar characteristics and which might have the same source of origin.

\section{Results and Discussion}

\subsection{Method Validation}

3.1.1. Linearity, $L O D$, and $L O Q$. The calibration curve was plotted by analyzing five increasing concentrations of amphetamine standard solutions and the linearity was observed by calculating the correlation coefficient $\left(R^{2}\right)$ value of the plot. The method showed a good linearity over the tested range as the $R^{2}$ value was calculated to be 0.997 . The equation for linear regression was attained to be $y=0.182 x-0.5443$. The LOD and LOQ values were calculated to be $0.44 \mu \mathrm{g} / \mathrm{mL}$ and $0.975 \mu \mathrm{g} / \mathrm{mL}$, respectively, which showed good sensitivity of the method.

3.1.2. Precision and Accuracy. The precision and accuracy of the GC method were determined by calculating the $\%$ relative standard deviations (\% RSDs) in case of intra- and interday analysis and it was calculated to be $<2 \%$ for both peak area and the retention time of the analyte which were within the prescribed limits given in the ICH guidelines. Percent recoveries were also calculated for the intra- and interday samples and were found to be in the range of $100 \pm 2 \%$ which were also within the acceptable limits of the ICH guidelines. The precision and accuracy data are given in Table 1.

3.1.3. Specificity. To determine the specificity of the method, methanol as blank was injected to the sample and analyzed using the method and the chromatogram obtained was compared to the chromatograms of calibration standards. No interferences were observed at the retention times of analytes and IS showing the good specificity of the method.

3.2. GC-MS Analysis. Using the developed GC-MS method, various constituents present in all tablet samples were separated within $30 \mathrm{~min}$ runtime. The area $\%$ values of all the constituents were calculated and the values more than $1 \%$ were reported. A total of 59 compounds were identified using the technique, which were present in one or more samples with area \% more than 1 . The names and area \% values of all the components present in 30 samples are summarized in Table 1S (Supplementary file). The major constituents which were common in most of the samples are shown in Table 2 and the structures thereof are depicted in Figure 1 along with their area \% values. Figures 2(a)-2(c) represent the total ion chromatograms for representative samples from each city. 
TABLE 1: Results of precision and accuracy experiments for intra- and interday analysis of the three quality control samples using the proposed GC method.

\begin{tabular}{lcccc}
\hline & Concentrations of samples $(\mu \mathrm{g} / \mathrm{mL})$ & \% RSD of peak area* & \% RSD of retention time* & \% recovery \pm SD $^{*}$ \\
\hline \multirow{3}{*}{ Intraday analysis } & 5.0 (LQC) & 0.75 & 0.92 & 99.4 \\
& 10.0 (MQC) & 0.63 & 0.54 & 0.88 \\
\hline \multirow{3}{*}{ Interday analysis } & 20.0 (HQC) & 0.94 & 0.63 & 98.2 \\
& 5.0 (LQC) & 0.36 & 0.49 & 98.2 \\
& 10.0 (MQC) & 0.57 & 0.53 & 98.6 \\
\hline
\end{tabular}

${ }^{*} n=3$; LQC: low quality control, MQC: medium quality control, and HQC: high quality control.

The tablet samples from Ahad Al-Masariha, Al-Darb, and Jazan showed considerable variations in their contents. Amphetamine was the only constituent that was detected at retention time (RT) of $4.78 \mathrm{~min}$ in all samples but in different area \% values ranging from 16.29 to $41.23 \%$ in all tablet samples. This variation was also observed in different samples from the same city. Another amphetamine derivative, $\mathrm{N}$-formyl amphetamine, was also present at RT of $8.15 \mathrm{~min}$ in few samples from each city in the range of $1.54-2.9 \%$, which might be present as an impurity that has been introduced during the manufacture of amphetamine or as a degradation product of amphetamine during storage. Another important constituent that was present in most of the samples was methyl diphenyl methyl ether at RT of $8.57 \mathrm{~min}$ in the range of $3.47-28.63 \%$ of the total constituents present.

Interestingly, several unusual compounds were also detected such as caffeine $(9.3-25.71 \%, \mathrm{RT}=10.88 \mathrm{~min})$ and lidocaine $(12.98-26.1 \%, \mathrm{RT}=11.03 \mathrm{~min})$ in good to significant amounts. Additionally, most of the samples showed presence of dimenhydrinate at RT $10.96 \mathrm{~min}$, which is a combination of diphenhydramine and 8-chlorotheophylline as $2.05-13.05 \%$ of the total constituents. $\mathrm{N}, \mathrm{N}^{\prime}$-di(phenyl isopropyl) formamide and di(phenyl isopropyl) amine were another two constituents which were present in considerable amounts with their area \% values ranging from 1.4 to $3.5 \%$ and from 1.4 to $21.5 \%$ with RT of $13.72 \mathrm{~min}$ and $11.13 \mathrm{~min}$, respectively. A number of long chain aliphatic alcohols were also detected in most of the samples including 2-octyldodecanol (1.9-8.65\%), 2-hexyldodecanol (1.6-9.98\%), and n-nonadecanol (1.04-2.51\%).

Few compounds were found to be specifically present in tablets collected from certain cities and were not common in all. For instance, 13-docosenamide was present at RT of $16.26 \mathrm{~min}$ in samples obtained from Al-Darb (2 out of 10) and Jazan (8 out of 10) cities only in the area \% values ranging from 1.99 to $2.93 \%$ but was absent in all the tested tablets from Ahad Al-Masariha city. Similarly, Cycloeicosane was also present in Al-Darb tablet samples $(1.87-2.63 \%)$ as well as tablets from Jazan (1.51-2.11\%) at RT of $11.47 \mathrm{~min}$ but not in Ahad Al-Masariha samples. Another constituent, heptacosane, was exclusively present at RT of $16.27 \mathrm{~min}$ in 5 out of 10 tested tablets from Al-Darb city in a considerable area \% values of $1.47-2.8 \%$, whereas it was absent in all samples from Ahad Al-Masariha and Jazan cities. All of the rest detected constituents either were present in very few samples or were present in very less area
$\%$ values. These were considered insignificant and may have arisen due to impurities from any of the ingredient at the time of manufacturing the tablets.

The illicit usage and peddling of amphetamine and related substances have been a cause of concern for most of the countries in the Middle Eastern and European regions. Seizure of illegal captagon tablets is common in this region and a number of cases have already been surfaced from many countries including Saudi Arabia, Yemen, Turkey, Iraq, Jordan, Serbia, and so forth and the number keeps counting. When subjected to forensic analysis, amphetamine was found to be the major constituent of these tablets with several other additives [17-23]. The amphetamine content in these studies was found to be very high and varied considerably among the samples. It was as low as $16 \%$ in some tablets and as high as $41 \%$ in others and tablets from all the cities showed the same variation. This indicated that there was not any fixed protocol used in the manufacture of these tablets and amphetamine was added to tablets randomly irrespective of its weight. Similar results were found previously in analyses of illegal captagon tablets in Saudi Arabia and Iraq, which revealed 2-38\% amphetamine content in all tablets [24]. Comparatively higher amount of amphetamine present in the tested tablets reveals the toxicity it can cause as amphetamine at this dose can cause a number of untoward reactions and dependence. High doses of amphetamine lead to impairment of cognitive functions and augmentation of nerve damage along with the induction of rapid muscles breakdown $[25,26]$.

The findings of this study were similar to the findings reported in an earlier study in which the presence of nonpsychoactive substances such as caffeine, quinine, ephedrine, paracetamol, theophylline, and chloroquine in $12-16 \%$ of the seized tablets from Saudi Arabia [27] was reported. Addition of theophylline derivatives such as 8chlorotheophylline to the tablets suggested that the manufacturer wanted to produce an effect similar to captagon tablets containing fenethylline [27], which is a theophylline derivative of amphetamine used in captagon tablets. Theophylline is a drug used to treat chronic obstructive pulmonary diseases (COPD) and asthma and is a known bronchodilator having positive inotropic and chronotropic effects. The theophylline derivatives are also known to stimulate the central nervous system similar to caffeine and these two substances must have been added to the tablets to increase the psychostimulant effects of amphetamine [27]. 


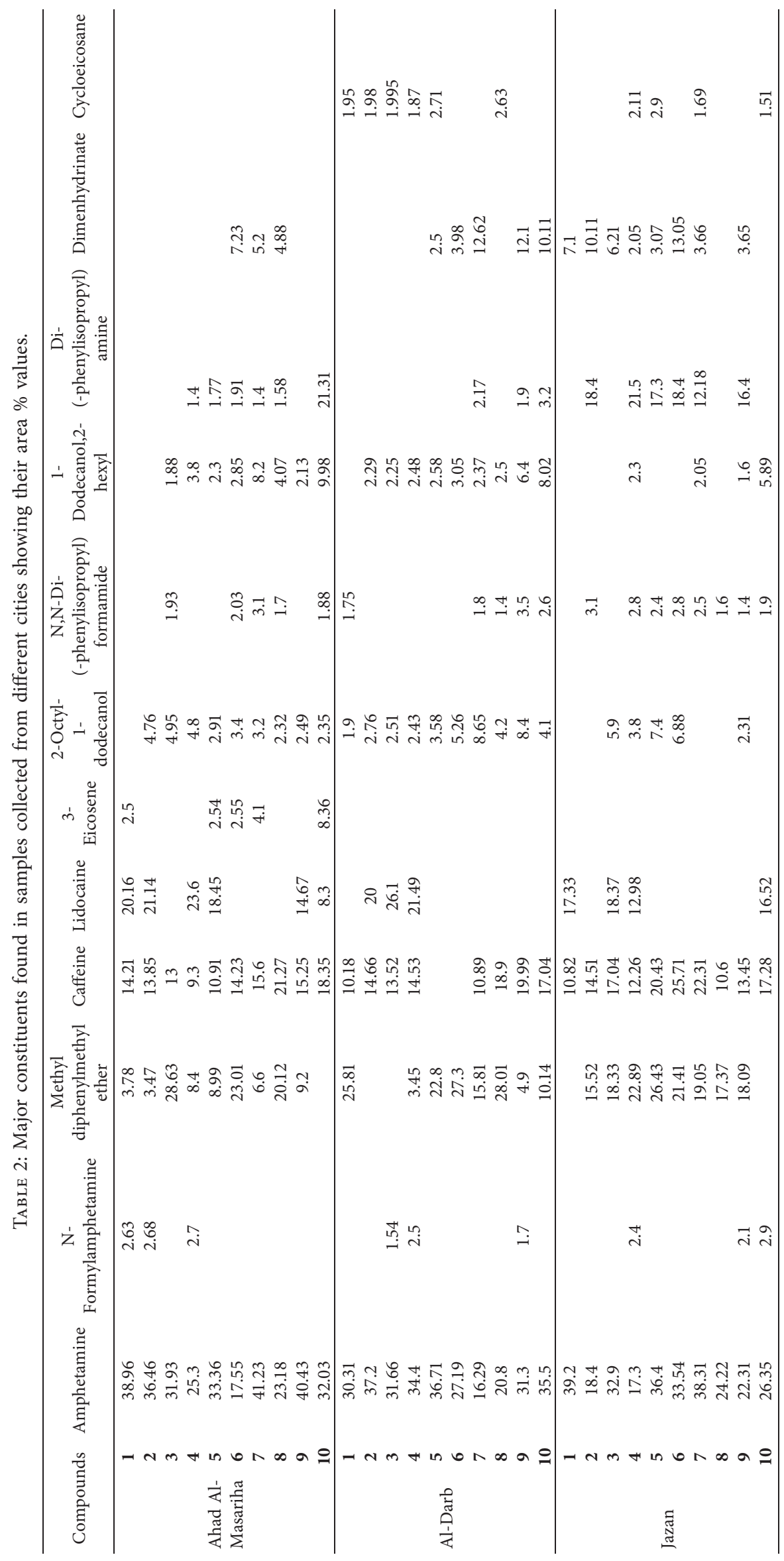


<smiles>CC(N)Cc1ccccc1</smiles>

Amphetamine (16.29-41.23\%)<smiles>Cn1c(=O)c2[nH]c(Cl)nc2n(C)c1=O</smiles>

\section{8-Chlorotheophylline} (2.05-13.05\%)<smiles>COC(c1ccccc1)c1ccccc1</smiles>

Methyl diphenyl ether $(3.47-28.63 \%)$

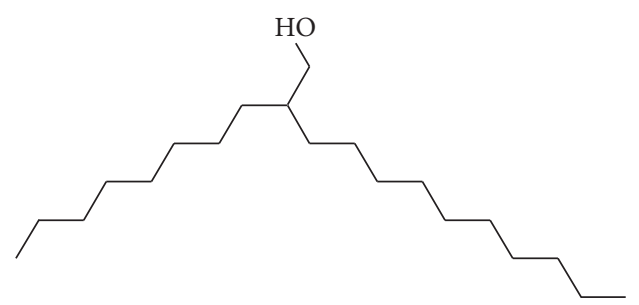

2-Octyl-dodecan-1-ol (1.9-8.65\%)<smiles>Cn1c(=O)c2c(ncn2C)n(C)c1=O</smiles>

Caffeine (9.3-25.71\%)

N,N-di (-phenylisopropyl) formamide<smiles>CC(C)(c1ccccc1)N(C=O)C(C)(C)c1ccccc1</smiles>
(1.4-3.5\%)

Diphenhydramine (2.05-13.05\%)<smiles>CN(C)CCOC(c1ccccc1)c1ccccc1</smiles><smiles>CC/C=C\CCCCCCCCCCCCCCCC</smiles>

3-Eicosene (2.5-8.36\%)<smiles>CCN(CC)CC(=O)Nc1c(C)cccc1C</smiles>

Lidocaine (12.98-26.1\%)

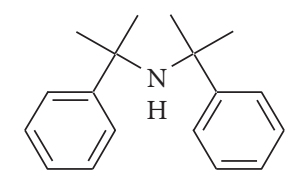

di- (-phenylisopropyl) amine $(1.4-21.5 \%)$

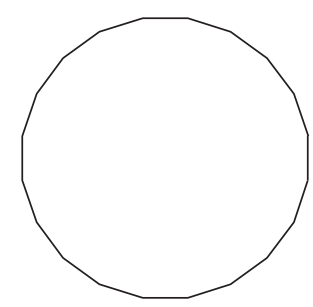

Cycloeicosane (1.51-2.9\%)

FIGURE 1: Structures of major constituents present in tablet samples showing their area \% values.

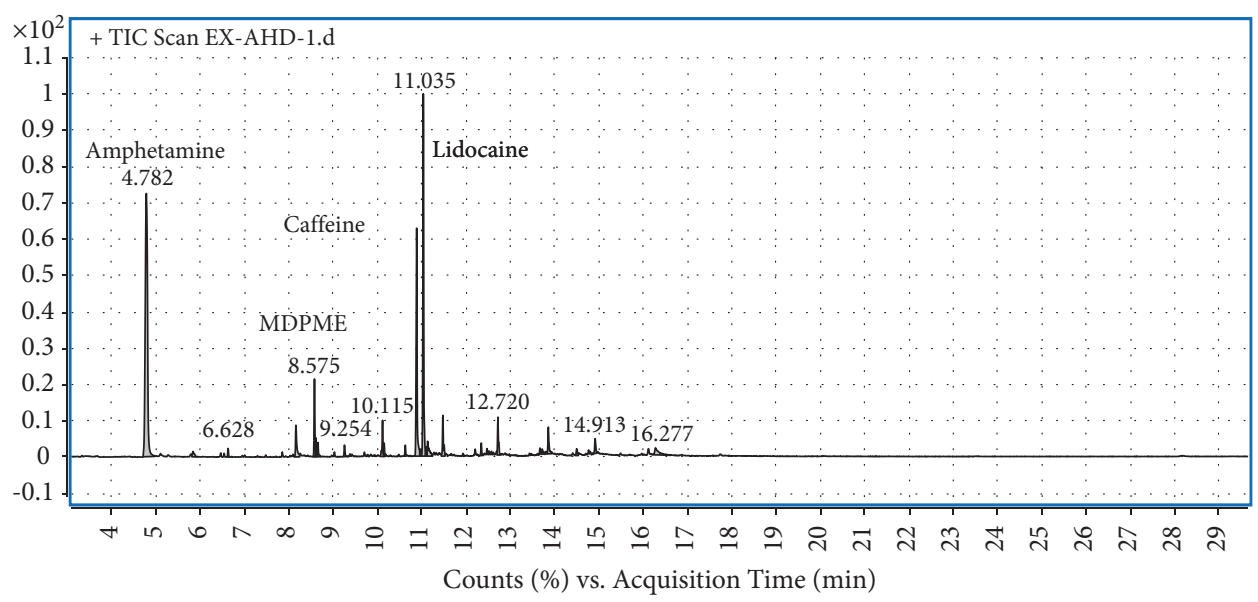

(a)

Figure 2: Continued. 


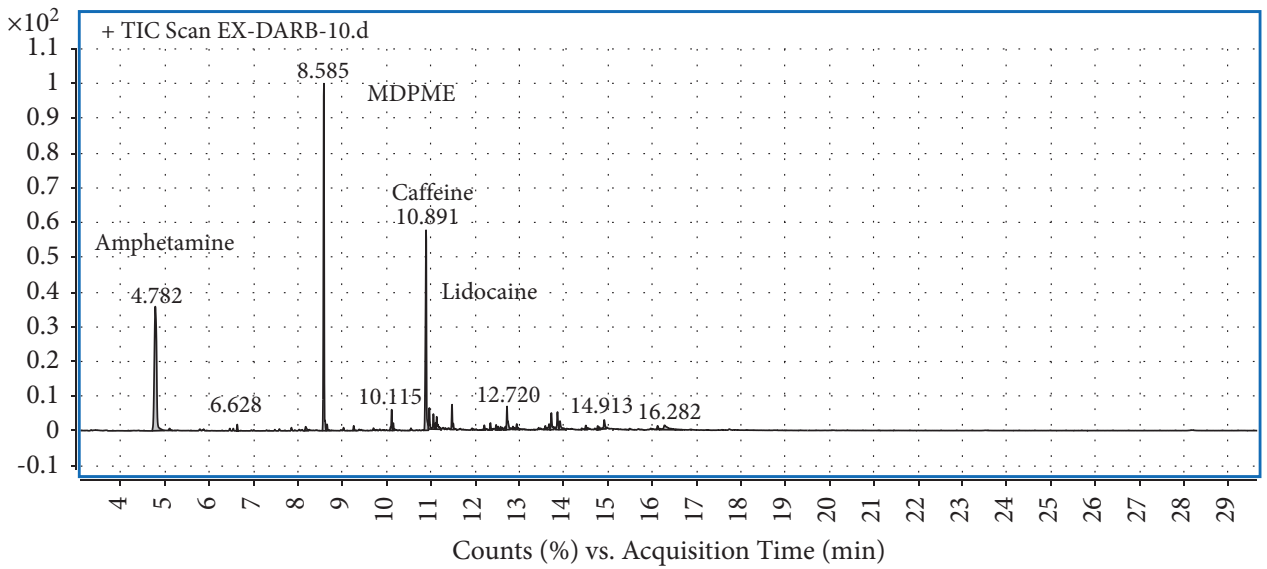

(b)

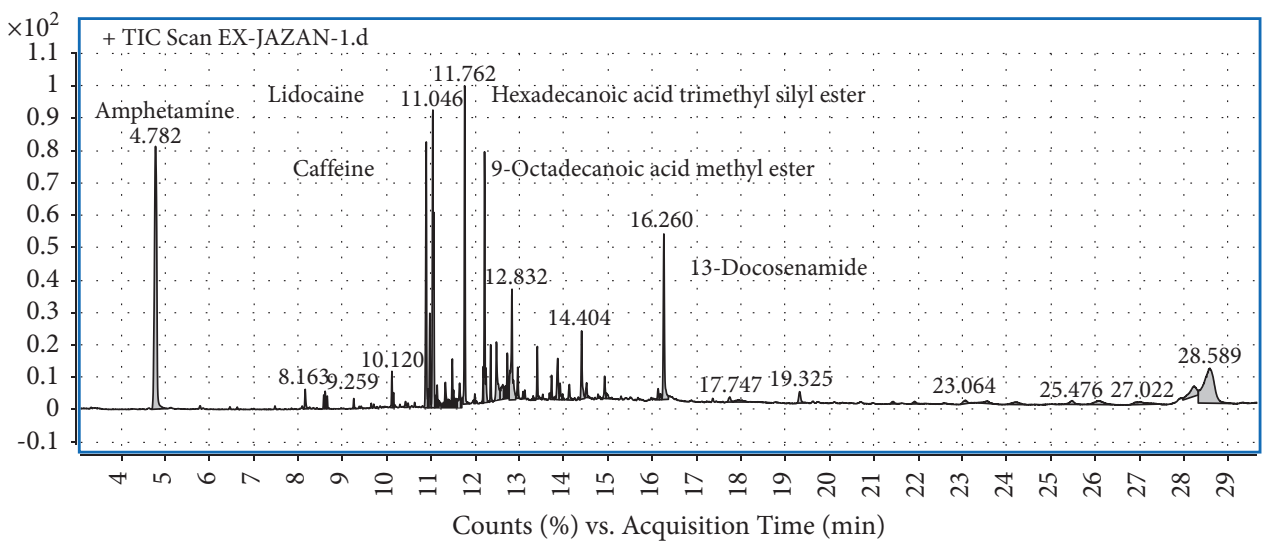

(c)

FIgURE 2: Total ion chromatograms (TIC) obtained for representative samples from each city. (a) TIC of A1, (b) TIC of D1, and (c) TIC of G1.

In a similar study, Alabdalla analyzed the counterfeit tablets of captagon seized from Jordan [17] using the GC-MS technique and detected amphetamine with several other additives including caffeine and quinine with the absence of fenethylline. Other seizures from Turkey [22] and Serbia [20] and subsequent analysis of tablets using GC-MS and FT-IR techniques revealed the presence of amphetamine along with other additives such as theophylline, caffeine, quinine, acetaminophen, ephedrine, lactose, diphenhydramine, and several other compounds, with absence of fenethylline. It suggested the similar trend of adding other compounds with amphetamine as in our study; most of the additives were the same and these additives are added to the amphetamine to enhance psychoactivity and, therefore, acceptability of the tablets.

The addition of diphenhydramine to the tablets was also important as diphenhydramine is a potent antihistaminic agent that is used therapeutically to treat allergic symptoms, common cold, and motion sickness. Additionally, it is also shown to have implications in neurotransmission affecting behavior including norepinephrine, dopamine, serotonin, opioid, and acetylcholine systems. It has also been investigated to have antianxiety, sedation, and antidepressant activities [28], which probably may have been the reason for its inclusion to the tablet. Finally, lidocaine was added for its nerve blocking activity leading to prevention of cardiovascular system side effects of amphetamines such as ventricular tachycardia and bleeding.

3.3. Hierarchical Cluster Analysis (HCA). Hierarchical Cluster Analysis (HCA) is one of the most common multivariate chemometric analyses used to determine the correlation between the constituents of different samples. It is employed to determine the hierarchy of clusters which are presented graphically as dendrogram or a tree. Samples are divided into pairs and the distances between these pairs are measured. These distances are the representative of dissimilarities between the sets. The variables are attached with each other in a hierarchical fashion in the dendrograms and the closest samples are considered to be most similar; however, the farthest is the most different [29]. Samples categorized in one cluster have unique and similar characteristics or, in other words, they are least dissimilar with each other and are separated by lesser distances. At each step, the two clusters that were most similar were joined into a single new cluster and once they get fused, objects were never separated. The two axes of dendrograms are the distance or the dissimilarities as the horizontal axis, whereas the samples and clusters are placed on the vertical axis. 


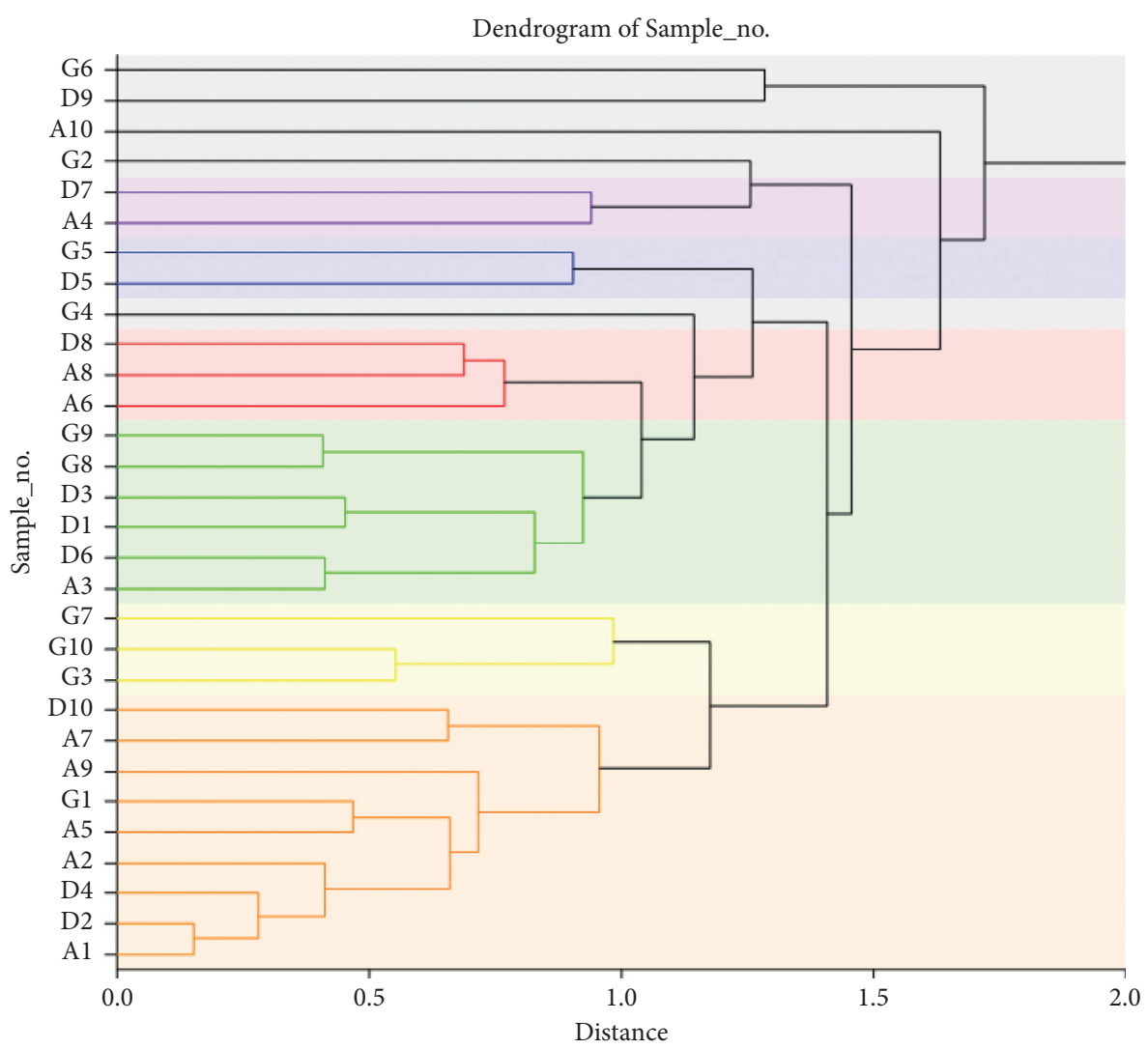

FIGURE 3: Dendrogram tree obtained for amphetamine tablet samples using average linkage (between groups) showing six clusters (colored lines) and distances between the samples.

When two clusters are fused, this is shown in the dendrogram, as the splitting of horizontal line to two horizontal lines and the horizontal position of the split signifies the dissimilarity (distance) between the two clusters.

All the 30 samples were classified into different clusters depending upon the constituents present in them which were considered as variables. Results are depicted as a dendrogram tree (Figure 3) which showed the presence of six distinct clusters of samples. If two samples were separated by a distance less than one, it was considered as significant and the lesser the distance was, the more was the similarity between the samples. The first cluster comprised three samples A6, A8, and D8, which showed similar characteristics as far as the constituents present in them are concerned. The second cluster consisted of two samples, D5 and G5, from Al-Darb and Jazan cities, respectively. The third cluster was having six samples, A3, D1, D3, D6, G8, and G9, consisting of samples from all three samples. The fourth cluster was again having two samples only, A4 and D7, from Ahad Al-Masariha and Al-Darb, respectively. The fifth cluster was the largest one having nine samples A1, A2, A5, A7, A9, D2, D4, D10, and G1 from all three cities. The sixth cluster comprised samples from Jazan city only showing presence of G3, G7, and G10 samples. The rest of all the samples G6, D9, A10, G2, and G4 were separated by distances more than 1.0 and were not significant, meaning that their characteristics are different from others and are linked to each other but not significantly.
Figure 3 shows dendrogram tree of the samples having all the samples connected to each other using colored lines. The black lines show distance more than 1 and hence, nonsignificant relations. Samples of fifth cluster showed most significant characteristics and are separated by orange lines and few samples are connected with a very short distance showing very close similarities between them. It was followed by samples from third cluster separated by green lines, sixth cluster separated by yellow lines, first cluster separated by red lines, second cluster separated by blue lines, and fourth cluster separated by violet line. Together they are connected at larger distances with nonsignificant black lines. The samples within the same cluster signify that they may have originated from the single source and together there may be six sources of samples tested in this study.

\section{Conclusions}

Using GC-MS technique, we were able to identify and quantify the constituents present in seized amphetamine tablets form three major cities of Jazan region, Saudi Arabia. A total of 30 samples were subjected to analysis using a simple and faster GCMS method and all the constituents were separated at different retention times. All the tablet samples showed amphetamine as the major constituent present in the range of $16.29-41.23 \%$ of the total constituents. Interestingly, other psychoactive and nonpsychoactive additives were also identified including caffeine, lidocaine, diphenhydramine, and 8-chlorotheophylline, 
which may have been added to enhance the effects of amphetamine and to increase the dependence. Several other organic compounds were also detected which altogether increase the toxicity of these tablets. Users as well as the government authorities should be informed and educated regarding the harmful constituents of these tablets which could help in stopping the illegal trafficking and use of these tablets on priority basis.

\section{Data Availability}

Data related to the study are available from the authors and can be produced upon request.

\section{Conflicts of Interest}

The authors declare that they have no conflicts of interest.

\section{Acknowledgments}

The authors wish to acknowledge the support provided by the Poison Control and Medical Forensic Chemistry Center, General Directorate of Health Affairs, Jazan, Saudi Arabia, for carrying out the analysis of unknown tablets.

\section{Supplementary Materials}

Table 1S: GC-MS analyses of amphetamine tablets collected from different cities of Jazan province. Ten tablets of amphetamine were analyzed in triplicate from three different cities, Ahad Al-Masariha, Al-Darb, and Jazan. (Supplementary Materials)

\section{References}

[1] S. M. Stahl, “Amphetamine (D, L)," Prescriber's Guide: Stahl's Essential Psychopharmacologypp. 45-51, Cambridge University Press, Cambridge, UK, 6th edition, 2017.

[2] Evekeo Prescribing Information (EPI) (2016) Arbor Pharmaceuticals LLC, 2019, https://www.evekeo.com/assets/ evekeo-pi.pdf/.

[3] European Monitoring Centre for Drugs and Drug Addiction, Captagon: Understanding Today's Illicit Market, EMCDDA Papers, Publications Office of the European Union, Luxembourg, Europe, 2018.

[4] M. Bassiony, "Substance use disorders in Saudi Arabia: review article," Journal of Substance Use, vol. 18, no. 6, pp. 450-466, 2013.

[5] N. Saquib, A. M. Rajab, J. Saquib, and A. AlMazrou, "Substance use disorders in Saudi Arabia: a scoping review," Substance Abuse Treatment, Prevention, and Policy, vol. 15, no. 41, p. $41,2020$.

[6] H. M. Al-Musa and S. D. S. Al-Montashri, "Substance abuse among male secondary school students in Abha city, Saudi Arabia: prevalence and associated factors," Biomedical Research, vol. 27, pp. 1364-1373, 2016.

[7] United Nations Office on Drugs and Crime (UNODC), Global Synthetic Drugs Assessment, Amphetamine-type Stimulants and New Psychoactive Substances, United Nations Office on Drugs and Crime, New York, NY, USA, 2014, https://www. unodc.org/documents/scientific/2014_Global_Synthetic_ Drugs_Assessment_web.pdf.
[8] M. Al-Mazroua, "Analysis of captagon tablets in Saudi Arabia, presentation to the ministry of health of Saudi Arabia," 2019, https://www.moh.gov.sa/en/Sectors/PCCs/DMM/PCC_Infor mation/Publications/Analysis\%20of\%20Captagon\%20Tablets \%20in\%20Saudi\%20Arabia.pdf.

[9] Drug Enforcement Administration (DEA), "Captagon mimic tablets (containing d,l-amphetamine, caffeine, theophylline, and other components) in Al Anbar Province, Iraq," Microgram Bull, vol. 42, no. 3, pp. 28-29, 2009.

[10] German Bundeskriminalamt (BKA), Captagon, Presentation, BKA (Germany), pp. 18-19, EMPACT Synthetic Drugs Meeting, Prague, Czech Republic, 2016.

[11] H. A. Alhazmi, W. Ahsan, M. Al Bratty et al., "Analysis of amphetamine and methamphetamine contents in seized tablets from Jazan, Saudi Arabia by liquid chromatographymass spectroscopy (LC-MS/MS) and chemometric techniques," Saudi Pharmaceutical Journal, vol. 28, no. 6, pp. 703-709, 2020.

[12] A. Khalid, H. A. Alhazmi, A. N. Abdalla et al., "GC-MS analysis and cytotoxicity evaluation of shammah (smokeless tobacco) samples of Jazan region of Saudi Arabia as promoter of cancer cell proliferation," Journal of Chemistry, vol. 2019, Article ID 3254836, 8 pages, 2019.

[13] H. A. Alhazmi, A. Khalid, S. Sultana et al., "Determination of phytocomponents of twenty-one varieties of smokeless tobacco using gas chromatography-mass spectroscopy (GCMS)," South African Journal of Chemistry, vol. 72, no. 1, pp. 47-54, 2019.

[14] M. Al Bratty, W. Ahsan, H. A. Alhazmi, I. M. Attafi, I. A. Khardali, and S. I. Abdelwahab, "Determination of trace metal concentrations in different parts of the khat varieties (Catha edulis) using inductively coupled plasma-mass spectroscopy technique and their human exposure assessment," Pharmacognosy Magazine, vol. 15, pp. 449-458, 2019.

[15] H. Alhazmi, W. Ahsan, I. Attafi et al., "Elemental profiling of smokeless tobacco samples using inductively coupled plasmamass spectrometry, their chemometric analysis and assessment of health hazards," Pharmacognosy Magazine, vol. 14, no. 58, pp. 587-596, 2018.

[16] Customs Laboratories European Network, "Recording of mass spectra of organic substances such as new psychoactive substances or designer drugs with a mass selective detector after gas-chromatographic separation, ILIADe code 403," 2021, https://ec.europa.eu/taxation_customs/sites/taxation/ files/iliade_403_gcms_drugs.pdf.

[17] M. A. Alabdalla, "Chemical characterization of counterfeit captagon tablets seized in Jordan," Forensic Science International, vol. 152, pp. 185-8, Article ID 185, 2005.

[18] N. Al-Gharably and A. R. Al-Obaid, "The characterization of counterfeit captagon tablets," Journal of the Forensic Science Society, vol. 34, no. 3, pp. 165-167, 1993.

[19] D. Dimova and N. Dinkof, "Psychotropic substances of the amfetamine type used by drug addicts in Bulgaria. Synthesis and medical forms," Analytical Methods of Identification, Scientific and Technical Notes, SCITEC/10, United Nations Office on Drugs and Crime, Sofia, Bulgaria, 1994.

[20] M. Nevescanin, S. Banovic-Stevic, S. Petrovic, and V. Vajs, "Analysis of amphetamines illegally produced in Serbia," Journal of the Serbian Chemical Society, vol. 73, no. 7, pp. 691-701, 2008.

[21] United Nations Office on Drugs and Crime (UNODC), World Drug Report 2009, United Nations Office on Drugs and Crime, New York, NY, USA, 2009. 
[22] Turkish Monitoring Centre for Drugs and Drug addiction (TUBIM), National Report (2012 Data) to the EMCDDA by the Reitox National Focal Point, Turkish Monitoring Centre for Drugs and Drug addiction, Ankara, Turkey, 2013.

[23] S. Demirkiran, C. Zeren, A. Çelikel, and M. M. Arslan, "The captagon tablets captured in Hatay between 2011-2012," Turkish Journal of Forensic Medicine, vol. 28, no. 1, pp. 17-23, 2014.

[24] European Academy of Forensic Science, "Istanbul Üniversitesi, \& European Academy of Forensic Science," Proceedings of the 3rd European Academy of Forensic Science Meeting, Istanbul, Turkey, Elsevier, 2003.

[25] M. Carvalho, H. Carmo, V. M. Costa et al., "Toxicity of amphetamines: an update," Archives of Toxicology, vol. 86, no. 8, pp. 1167-1231, 2012.

[26] S. Berman, J. O’Neill, S. Fears, G. Bartzokis, and E. D. London, "Abuse of amphetamines and structural abnormalities in the brain," Annals of the New York Academy of Sciences, vol. 1141, no. 1, pp. 195-220, 2008.

[27] Drug enforcement administration (DEA) (2003) fenethylline and the middle east: a brief summary, DEA-03046.

[28] É. Lessard, M.-A. Yessine, B. A. Hamelin et al., "Diphenhydramine alters the disposition of venlafaxine through inhibition of CYP2D6 activity in humans," Journal of Clinical Psychopharmacology, vol. 21, no. 2, pp. 175-184, 2001.

[29] P. M. Mather, Computational Methods of Multivariate Analysis in Physical Geography, John Wiley \& Sons, London, UK, 1976. 